\title{
Eigenvalue Pairing in the Response Matrix for a Class of Network Models with Circular Symmetry *
}

\author{
Miriam Farber \\ Department of Mathematics \\ Technion - Israel Institute of Technology \\ Haifa, Israel \\ miriamf@tx.technion.ac.il
}

\author{
Charles R. Johnson \\ Department of Mathematics \\ Collage of William and Mary \\ Virginia, U.S.A. \\ crjohnso@math.wm.edu
}

\author{
Wei Zhen \\ Department of Mathematics \\ Imperial College \\ London, United Kingdom \\ zhen.wei11@imperial.ac.uk
}

Submitted: April 24, 2013; Accepted: July 28, 2013; Published: Aug 9, 2013

Mathematics Subject Classifications: 05C50, 15A18, 90B10, 05C10

\begin{abstract}
We consider the response matrices in certain weighted networks that display a circular symmetry. It had been observed empirically that these exhibit several paired (multiplicity two) eigenvalues. Here, this pairing is explained analytically for a version of the model more general than the original. The exact number of necessarily paired eigenvalues is given in terms of the structure of the model, and the special structure of the eigenvectors is also described. Examples are provided.
\end{abstract}

Keywords: circular planar graph; Dirichlet-to-Neumann matrix; eigenvalue pairing; resistor network

\section{Introduction}

We begin by describing the physical model underlying our work, using the definitions and notations from $[5,7]$, see also [4]:

\footnotetext{
*This work supported by NSF/DMS grant number DMS-0751964
} 
A graph with boundary is a triple $G=\left(V, V_{B}, E\right)$, in which $V$ is the set of nodes, $E$ is the set of edges, and $V_{B}$ is a nonempty subset of $V$ called the set of boundary nodes. The set $I=V \backslash V_{B}$ is called the set of interior nodes.

A circular planar graph is a graph $G$ with boundary that is embedded in a disc $D$ in the plane so that the boundary nodes lie on the circle $C$ which bounds $D$, and the rest of $G$ is in the interior of $D$. The boundary nodes are labeled $v_{1}, \ldots, v_{n}$ in clockwise order around $C$.

A conductivity on a graph $G$ is a function $\gamma$ that assigns to each edge $e$ in $G$ a positive real number $\gamma(e)$, called the conductance of the edge $e$. Finally, a resistor network $\Gamma=(G, \gamma)$ is a graph $\mathrm{G}$ together with a conductivity function $\gamma$.

If $\Gamma$ is a resistor network with boundary, the set $V_{B}$ of boundary nodes is sometimes denoted $\partial G$, and the set $I=V \backslash V_{B}$ of interior nodes of $G$ is sometimes denoted int $G$.

Let $\Gamma=(G, \gamma)$ be a connected resistor network, and let $u$ be the voltage function defined at all the nodes of $G$. Then $u$ is called $\gamma$-harmonic if for any node $w \in \operatorname{int} G$, $\sum_{q \sim w} \gamma(\{w, q\})(u(w)-u(q))=0$. For a network $\Gamma=(G, \gamma)(G$ has $m$ nodes) with a $\gamma$-harmonic voltage function $u$, the Kirchhoff matrix is an $m$-by- $m$ matrix $K$ defined as follows:

$$
K_{i j}=\left\{\begin{array}{cl}
0 & \text { if }\{i, j\} \notin E(G) \\
-\gamma(\{i, j\}) & \text { if }\{i, j\} \in E(G) \\
\sum_{q \neq i} \gamma(\{i, q\}) & \text { if } i=j
\end{array}\right.
$$

Using $K$, the resulting current flow into $\Gamma$ is $\phi=K u$. One can view $K$ as

$$
K={ }_{i n t G}^{\partial G}\left(\begin{array}{cc}
\partial G & i n t G \\
A & B \\
B^{T} & C
\end{array}\right)
$$

If $\Gamma$ is a connected resistor network with boundary, the response matrix (which is also called Dirichlet-to-Neumann matrix) $\Lambda_{\gamma}$ can be obtained as the Schur complement $[6,9]$ in $K$ of the square submatrix corresponding to the interior nodes of $\Gamma: \Lambda_{\gamma}=A-B C^{-1} B^{T}$. Let $\varphi$ and $\psi$ be column vectors such that $\varphi_{i}$ is the boundary voltage and $\psi_{i}$ is the boundary current at a boundary node $i$. Then one can compute $\psi$ using the response matrix by $\psi=\Lambda_{\gamma} \varphi$.

In analyzing the response matrix of certain resistor networks, the third author empirically noticed that it displayed several eigenvalues of multiplicity two, independent of $\gamma$. These networks (described in section 2) involve a circular symmetry that implies equality in certain edge weights, but the eigenvalue pairing is unusual compared to such models in general. It is our purpose here to explain this eigenvalue pairing analytically, and in the process, to make observations regarding the structure of the eigenvectors of the response matrix. We also note limitations on the occurrence of this eigenvalue pairing. Eigenvalues of the response matrix of certain types of networks have been discussed widely in the past (e.g. $[3,5,8]$ ). Such information as pairing can only improve the ability to calculate eigenvalues of such response matrices. 
In the next section we describe the model, its Kirchhoff matrix (which is actually the Laplacian matrix of the graph) and the resulting response matrix (a Schur complement in the Laplacian). Necessary background and comments are also given. Then, in the third section, we prove the main theorem that explains the eigenvalue pairing. In the fourth section, we generalize the results to a much wider family of networks. Along the way, further structural observations are made about the eigenvectors.

\section{The Model}

The resistor networks we consider may be described by a weighted undirected, planar graph with a single center vertex, surrounded by concentric circles, with N "long" radial lines emanating the same distance out ( at least to the last circle) from the center and $\mathrm{S}$ "short" radial lines. Both form vertices when they intersect the concentric circles. The short lines begin on circles and extend outward; there is the same number $\mathrm{B}$ of short lines between each pair of consecutive long lines. Each such collection is required to be right-left symmetric and the same, in order, as the next. We call such a graph circular symmetric (note that the underlying graph is a circular planar graph). As an example, consider Figure 1

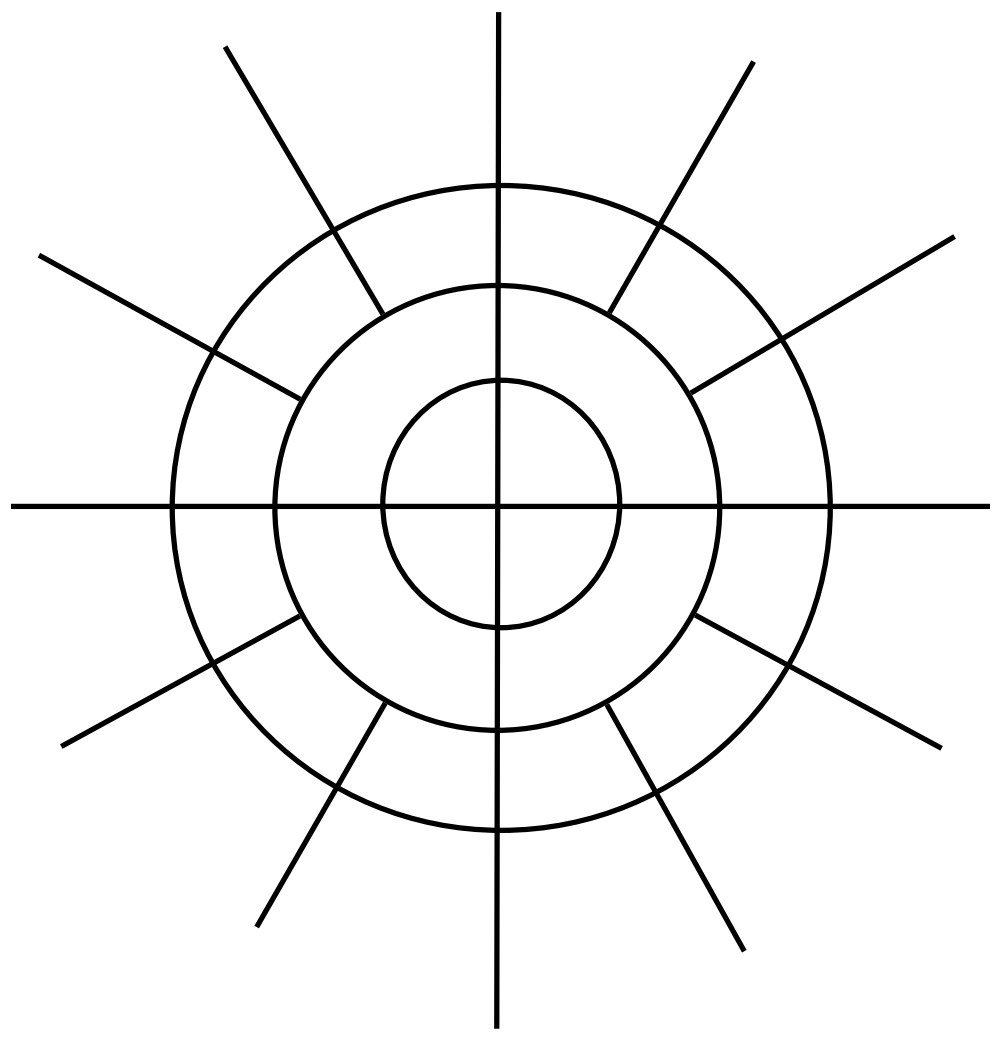

Figure 1: A circular symmetric graph 
In this case $N=4$ and $B=2$, so that $S=8$. We assume that the weights on the edges are related as presented in Figure 2.

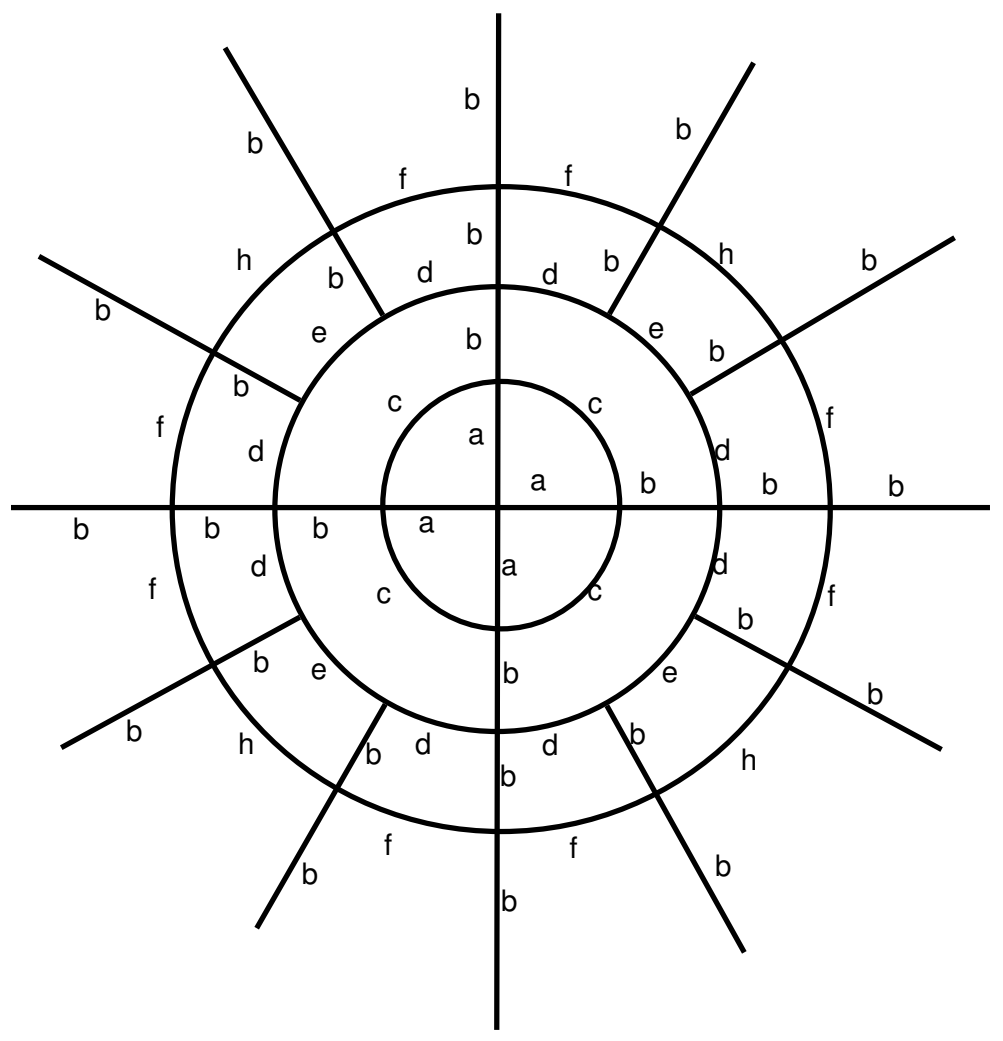

Figure 2: A weighted circular symmetric graph

All edge weights are assumed positive with no other conditions or symmetries assumed. We call a circular symmetric graph, with weights as above "properly weighted". All edge weights, for edges between two consecutive concentric circles (or beyond the last one), are the same, but this weight may differ, depending upon the pair of consecutive concentric circles. For edges on a concentric circle, between two consecutive long lines, right-left symmetry is required, as well as the same weights between the next pair of long lines.

Consider another example presented in Figure 3

The weighted Laplacian of a circular symmetric graph, properly weighted, is then the following singular M-matrix. Since the graph is connected, it has rank deficiency of 1. 


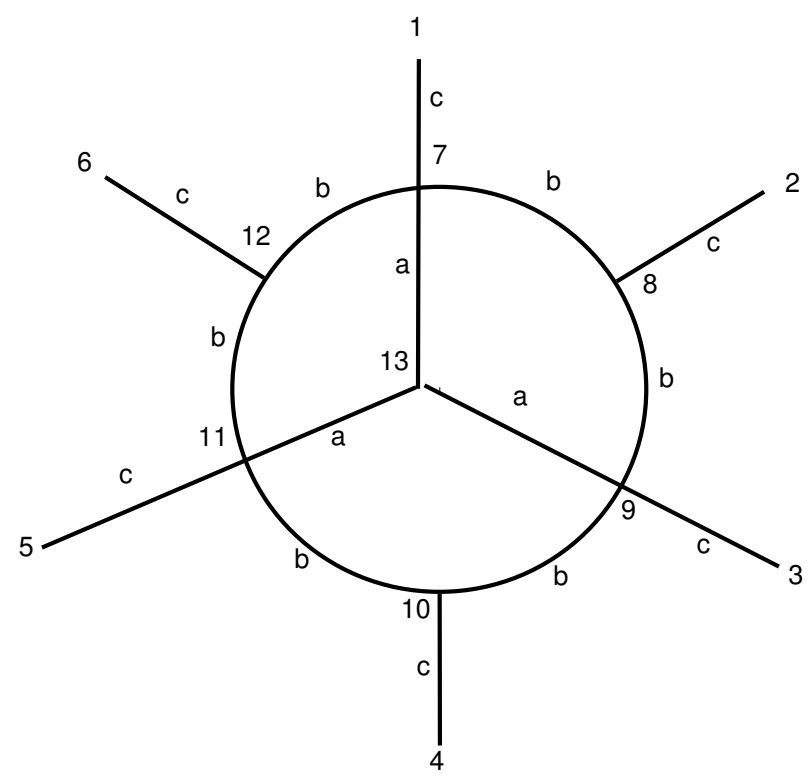

Figure 3: Second example of weighted circular symmetric graph

$$
L=\left(\begin{array}{ccccccccccccc}
c & 0 & 0 & 0 & 0 & 0 & -c & 0 & 0 & 0 & 0 & 0 & 0 \\
0 & c & 0 & 0 & 0 & 0 & 0 & -c & 0 & 0 & 0 & 0 & 0 \\
0 & 0 & c & 0 & 0 & 0 & 0 & 0 & -c & 0 & 0 & 0 & 0 \\
0 & 0 & 0 & c & 0 & 0 & 0 & 0 & 0 & -c & 0 & 0 & 0 \\
0 & 0 & 0 & 0 & c & 0 & 0 & 0 & 0 & 0 & -c & 0 & 0 \\
0 & 0 & 0 & 0 & 0 & c & 0 & 0 & 0 & 0 & 0 & -c & 0 \\
-c & 0 & 0 & 0 & 0 & 0 & c+2 b+a & -b & 0 & 0 & 0 & -b & -a \\
0 & -c & 0 & 0 & 0 & 0 & -b & c+2 b & -b & 0 & 0 & 0 & 0 \\
0 & 0 & -c & 0 & 0 & 0 & 0 & -b & c+2 b+a & -b & 0 & 0 & -a \\
0 & 0 & 0 & -c & 0 & 0 & 0 & 0 & -b & c+2 b & -b & 0 & 0 \\
0 & 0 & 0 & 0 & -c & 0 & 0 & 0 & 0 & -b & c+2 b+a & -b & -a \\
0 & 0 & 0 & 0 & 0 & -c & -b & 0 & 0 & 0 & -b & c+2 b & 0 \\
0 & 0 & 0 & 0 & 0 & 0 & -a & 0 & -a & 0 & -a & 0 & 3 a
\end{array}\right)
$$

The response matrix for this network model, which we denote by $R$, is simply the Schur complement of the diagonal principle submatrix $L[7,8,9,10,11,12,13 \mid 7,8,9,10,11,12,13]$ in $L$. If $a=1, b=2$ and $c=3$, then in this case we get

$$
R=\left(\begin{array}{cccccc}
\frac{445}{286} & -\frac{137}{286} & -\frac{133}{572} & -\frac{19}{143} & -\frac{133}{572} & -\frac{137}{286} \\
-\frac{137}{286} & \frac{206}{143} & -\frac{137}{286} & -\frac{25}{143} & -\frac{19}{143} & -\frac{25}{143} \\
-\frac{133}{572} & -\frac{137}{286} & \frac{445}{286} & -\frac{137}{286} & -\frac{133}{572} & -\frac{19}{143} \\
-\frac{19}{143} & -\frac{25}{143} & -\frac{137}{286} & \frac{206}{143} & -\frac{137}{286} & -\frac{25}{143} \\
-\frac{133}{572} & -\frac{19}{143} & -\frac{133}{572} & -\frac{137}{286} & \frac{445}{286} & -\frac{137}{286} \\
-\frac{137}{286} & -\frac{25}{143} & -\frac{19}{143} & -\frac{25}{143} & -\frac{137}{286} & \frac{206}{143}
\end{array}\right)
$$


Because of the inertia theorem for Schur complements [9], this matrix is necessarily (symmetric) positive semidefinite with just one zero eigenvalues. It is the remaining (positive) eigenvalues in which we are interested. In general, the response matrix may be written as $R=A-B C^{-1} B^{T}$, when $L=\left(\begin{array}{cc}A & B \\ B^{T} & C\end{array}\right)$ is the weighted Laplacian, such that the rows (and columns) of $A$ correspond to the vertices of degree one in the circular symmetric graph.

As we shall see, the response matrix necessarily has several multiplicity two "paired") eigenvalues. Aside from the pairing, we have not found these eigenvalues to exhibit any other particular structure. For example, the eigenvalues of the matrix $R$ from above are $\left\{\frac{24}{11}, \frac{3}{104}(59+3 \sqrt{17}), \frac{3}{104}(59+3 \sqrt{17}), \frac{3}{104}(59-3 \sqrt{17}), \frac{3}{104}(59-3 \sqrt{17}), 0\right\}$, and one can see that there are two eigenvalues of multiplicity two.

In the next section, we describe how many paired eigenvalues must appear in $R$ and why, in our main theorem. In the process, special structure in the eigenvectors is mentioned. Examples show that, in general, the number of proven pairs is best possible.

\section{The number of paired eigenvalues in $R$}

The two key parameters in the network model are $\mathrm{N}$, the number of long radial lines extending from the center vertex and the number $\mathrm{S}$ of shorter radial lines not incident with center. There is a fixed number $B$ of these shorter lines between each pair of longer lines, so that $S=B N$. The total $A=N+S$ of these lines is then $(B+1) N$. Let U be the number of long lines extending strictly above the horizontal axis when one of the long lines determines the horizontal axis and the remainder are evenly spaced. So, $U=\frac{N-1}{2}$ or $\frac{N-2}{2}$ depending on whether $\mathrm{N}$ is odd or even. Then, the minimum number of paired eigenvalues in the response matrix is as follows.

Theorem 1. Let $R$ be the response matrix of a properly weighted, circular symmetric network. Then, with the parameters defined above, $R$ has at least

$$
(B+1) U
$$

paired eigenvalues.

Remark 2. It may happen that two paired eigenvalues are the same, making a multiplicity 4 eigenvalue, for example. But generically there will $(B+1) U$ distinct paired eigenvalues and no more. So, an alternate statement would be that there are at least $2(B+1) U$ eigenvalues counting multiplicity, of positive even multiplicity.

Before presenting the proof, we introduce several notations. Let $\alpha, \beta \in \mathrm{N}, \alpha \geqslant 2$. 
Define the matrix $T_{\alpha, \beta}$ of order $\alpha, \beta$ in the following way

$$
T_{\alpha, \beta}=\left[\begin{array}{ccccc}
0 & I_{\beta} & 0 & \ldots & 0 \\
0 & 0 & I_{\beta} & \ldots & 0 \\
\vdots & \vdots & \vdots & \ddots & \vdots \\
0 & 0 & 0 & \ldots & I_{\beta} \\
I_{\beta} & 0 & 0 & 0 & \ldots
\end{array}\right]
$$

such that $I_{\beta}$ appears $\alpha$ times in total. For example, for $\alpha=4, \beta=3$ we have:

$$
T_{4,3}=\left[\begin{array}{cccc}
0 & I_{3} & 0 & 0 \\
0 & 0 & I_{3} & 0 \\
0 & 0 & 0 & I_{3} \\
I_{3} & 0 & 0 & 0
\end{array}\right]
$$

We denote by $C_{n}$ the anti-diagonal matrix of order $n$, all of those anti-diagonal entries are ones. The matrix $P_{n}$ is defined in the following way:

$$
P_{n}=\left[\begin{array}{cc}
1 & 0 \\
0 & C_{n-1}
\end{array}\right]
$$

For example,

$$
P_{5}=\left[\begin{array}{lllll}
1 & 0 & 0 & 0 & 0 \\
0 & 0 & 0 & 0 & 1 \\
0 & 0 & 0 & 1 & 0 \\
0 & 0 & 1 & 0 & 0 \\
0 & 1 & 0 & 0 & 0
\end{array}\right]
$$

We are now ready to present the proof of Theorem 1 :

Proof. Using the symmetries of a properly weighted graph, as well as known relations between matrices of the form $P_{A}$ and $T_{N, B+1}$, we get:

$$
\begin{aligned}
T_{N, B+1} R & =R T_{N, B+1} \\
P_{A} R & =R P_{A} \\
T_{N, B+1} P_{A} & =P_{A} T_{N, B+1}^{N-1}
\end{aligned}
$$

It is well known that the eigenvalues of matrices of the form $T_{N, B+1}$ are

$$
\left\{1^{(B+1)}, w^{(B+1)}, w^{2^{(B+1)}}, \ldots, w^{N-1(B+1)}\right\}
$$


where $w$ is a primitive $N^{t h}$ root of unity, and the brackets indicate the multiplicity. Note that $T_{N, B+1}$ is a permutation matrix and $\mathrm{R}$ is symmetric, and hence both of them are diagonalizable. In addition, according to (1), $T_{N, B+1}$ and $\mathrm{R}$ commute, and hence they

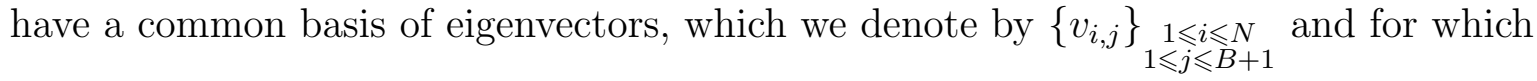

$$
\begin{gathered}
T_{N, B+1} v_{i, j}=w^{i-1} v_{i, j} \\
R v_{i, j}=\alpha_{i, j} v_{i, j}
\end{gathered}
$$

where $\left\{\alpha_{i, j}\right\}_{\substack{1 \leqslant i \leqslant N \\ 1 \leqslant j \leqslant B+1}}$ is the set of the eigenvalues of R. Using (4) and (3) and we get

$$
T_{N, B+1} P_{A} v_{i, j}=P_{A} T_{N, B+1}^{N-1} v_{i, j}=w^{(i-1)(N-1)} P_{A} v_{i, j}
$$

for all $1 \leqslant i \leqslant N, 1 \leqslant j \leqslant B+1$ Thus, we get that $P_{A} v_{i, j}$ is an eigenvector of $T_{N, B+1}$ that is associated with the eigenvalue $w^{(i-1)(N-1)}$. Let us assume from now on that $1<i \leqslant N$. Then

$$
w^{(i-1)(N-1)}=w^{i N-N-i+1}=w^{i N} w^{-N} w^{-i+1}=w^{-N} w^{-i+1}=w^{N} w^{-i+1}=w^{N-(i-1)}
$$

Therefore, since $P_{A} v_{i, j}$ is an eigenvector of $T_{N, B+1}$ with the eigenvalue $w^{N-(i-1)}$, then by (4) we have

$$
P_{A} v_{i, j} \in \operatorname{span}\left\{v_{N-i+2,1}, v_{N-i+2,2}, \ldots, v_{N-i+2, B+1}\right\}
$$

Note that since we assumed $i>1, v_{N-i+2, j}$ is defined. Hence, according to (5), we may write

$$
P_{A} v_{i, j}=\sum_{k=1}^{B+1} c_{i, j, k} v_{N-i+2, k}
$$

where not all the $c_{i, j, k}$ are zeros.

Therefore, from (6) and (4), we get

$$
R P_{A} v_{i, j}=\sum_{k=1}^{B+1} c_{i, j, k} \alpha_{N-i+2, k} v_{N-i+2, k}
$$

On the other hand, from (6) and (4) we have

$$
P_{A} R v_{i, j}=P_{A} \alpha_{i, j} v_{i, j}=\alpha_{i, j} P_{A} v_{i, j}=\sum_{k=1}^{B+1} \alpha_{i, j} c_{i, j, k} v_{N-i+2, k}
$$

Since by (2), $R P_{A} v_{i j}=P_{A} R v_{i, j}$ and $\left\{v_{i, j}\right\}$ are linearly independent, the coefficients of $v_{N-i+2, k}$ in (7) and (8) must be equal, and hence, for all $1 \leqslant k \leqslant B+1, c_{i, j, k} \alpha_{N-i+2, k}=$ $\alpha_{i, j} c_{i, j, k}$.

As we mentioned before, there exists $k$ for which $c_{i, j, k} \neq 0$. Dividing the appropriate equation by $c_{i, j, k}$ leads us to $\alpha_{N-i+2, k}=\alpha_{i, j}$ Therefore, if $i \neq N-i+2$, we get that 
$\alpha_{i, j}$ is of multiplicity at least 2. In order to avoid double counting, let us assume that $i<N-i+2$, and hence $2 i<N+2$, so $i<\frac{N+2}{2}$. Together with our original assumption, $1<i<\frac{N+2}{2}$. Hence, there are 2 cases:

$\mathrm{N}$ is odd, and $i \in\left\{2,3,4, \ldots, \frac{N+1}{2}\right\}$,

$\mathrm{N}$ is even, and $i \in\left\{2,3,4, \ldots, \frac{N}{2}\right\}$.

In both cases, $j \in\{1,2, \ldots, B+1\}$.

Note that for odd $N,\left|\left\{2,3, \ldots \frac{N+1}{2}\right\}\right|=U$, and similarly, for even $\mathrm{N},\left|\left\{2,3, \ldots \frac{N}{2}\right\}\right|=$ $U$. Therefore, in any event, we get that there are at least $U(B+1)$ pairs of equal eigenvalues.

\section{Further discussion and Generalization}

As one can see from the proof of our main result, we used only two properties of the network, which are equations (1) and (2). Thus, we want the network (including its weights) to be symmetric with respect to the $y$-axis, as well as preserve certain rotational symmetry (that depends on the parameters in (1)). Thus, using the notations from the beginning of section 3, as well as the same proof, we obtain the following:

Theorem 3. Let $R$ be the response matrix of a network satisfying (1) and (2). Then, with the parameters defined at the beginning of section $3, R$ has at least

$$
(B+1) U
$$

paired eigenvalues.

There are various examples of such networks. With proper weights, the networks in Figure 4 satisfy the theorem.
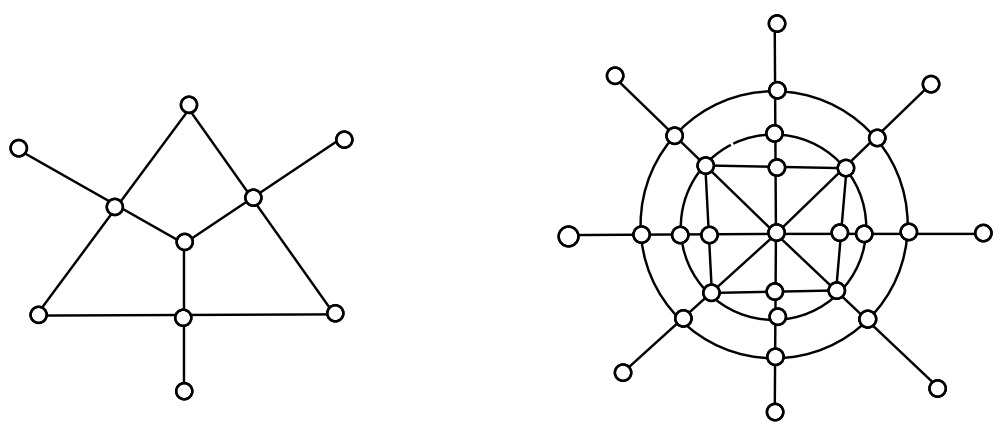

Figure 4: Networks that satisfy Theorem 3 


\section{Acknowledgements}

The authors would like to thank Cristina Arauz, Andres Encinas and Angeles Carmona, for their very valuable comments, in the preparation of this manuscript. By chance they have also been studying such networks and found the above pairing results of interest. See references [1, 2]; they also provided 9 pages of helpful notes.

\section{References}

[1] C. Arauz, A. Carmona and A. M. Encinas. Green function on product networks. VIII Jornadas de Matemaatica Discreta y Algoritmica, 2012, submitted.

[2] E. Bendito, A. Carmona, A. M. Encinas. Potential Theory for Schrodinger operators on finite netowrks. Rev. Mat. Iberoamericana, 21:771-818, 2005.

[3] O. D. Biesel, D. V. Ingerman, J. A. Morrow and W. T. Shore. Layered Networks, the Discrete Laplacian, and a Continued Fraction Identity. http://www.math.washington.edu/ reu/papers/current/william/layered.pdf.

[4] Y. Colin de Verdiere, I. Gitler, and D. Vertigan. Reseaux electriques planaires II. Comment. Math. Helv., 71(1):144-167, 1996.

[5] E. Curtis and J. Morrow. Inverse Problems for Electrical Networks. volume 13 of Series on Applied Mathematics, World Scientific, 2000.

[6] R. Horn, C. R. Johnson. Matrix analysis. Cambridge University Press, 1985.

[7] D. Ingerman, E. B. Curtis and J. Morrow. Circular planar graphs and resistor networks. Linear Algebra and its Applications, 283:115-150,1998.

[8] D. V. Ingerman. Discrete and Continuous Dirichlet-to-Neumann Maps in the Layered Case. SIAM Journal on Mathematical Analysis, 31:1214-1234,2000.

[9] F. Zhang. The Schur Complement and Its Applications. in Series Numerical Methods and Algorithms, Vol. 4, Springer, 2005. 